\title{
EUS-guided gall bladder drainage with a lumen-apposing metal stent: a prospective long-term evaluation
}

\author{
Daisy Walter, ${ }^{1}$ Anthony Y Teoh, ${ }^{2}$ Takao Itoi, ${ }^{3}$ Manuel Pérez-Miranda, ${ }^{4}$ \\ Alberto Larghi, ${ }^{5}$ Andres Sanchez-Yague, ${ }^{6}$ Peter D Siersema, ${ }^{1}$ Frank P Vleggaar ${ }^{1}$
}

- Additional material is published online only. To view please visit the journal online (http://dx.doi.org/10.1136/ gutjnl-2015-309925).

${ }^{1}$ Department of Gastroenterology and Hepatology, University Medical Center Utrecht, Utrecht, The Netherlands

${ }^{2}$ Department of Surgery, Chinese University of Hong Kong, Prince of Wales Hospital, Hong Kong, Hong Kong

${ }^{3}$ Department of

Gastroenterology and Hepatology, Tokyo Medical University, Tokyo, Japan

${ }^{4}$ Department of

Gastroenterology and Hepatology, University Hospita Rio Hortega, Valladolid, Spain ${ }^{5}$ Digestive Endoscopy Unit, Catholic University, Rome, Italy ${ }^{6}$ Department of Gastroenterology and Hepatology, Hospital Costa del Sol, Marbella, Spain

\section{Correspondence to} Daisy Walter, Department of Gastroenterology and Hepatology, University Medical Center Utrecht, P.O. Box 85500, Utrecht 3508 GA, The Netherlands;

d.walter@umcutrecht.nl

Received 6 May 2015 Accepted 8 May 2015 Published Online First 3 June 2015

\section{CrossMark}

To cite: Walter D, Teoh AY, Itoi T, et al. Gut 2016;65:68.
Endoscopic ultrasound-guided gall bladder drainage (EUS-GBD) has been shown to be comparable with percutaneous gall bladder drainage (PTGBD) in terms of technical feasibility and clinical efficacy for the treatment of acute cholecystitis in high-risk surgical patients. ${ }^{1}$ However, a potential serious complication of this technique is air or bile leakage into the peritoneal cavity, since insertion of a drain or plastic stent requires a fistula tract with a diameter larger than the diameter of the inserted drain or stent. Therefore, a specifically designed lumen-apposing metal stents (LAMSs) has been developed for transenteric drainage and successfully tested in animal models. ${ }^{2}{ }^{3}$ Preliminary clinical experience with LAMSs for drainage of peri-pancreatic fluid collections (PFCs) appears to be consistent with anchoring features tested in animal models. ${ }^{4-6}$ However, reports on the use of LAMSs for gall bladder drainage are limited to case reports and small case series without long-term follow-up. ${ }^{3} 5$ 7-12

We performed a multicentre, prospective study to determine the feasibility and safety of the use of LAMS for EUS-GBD in high-risk surgical patients with acute cholecystitis. A total of 30 patients were included. Technical success was achieved in 27 of 30 patients (90\%) (figure 1) and clinical success in 26 of 27 patients (96\%). Two of 27 patients (7\%) developed recurrent cholecystitis due to LAMS obstruction. Successful LAMS removal was performed in 15 of 30 patients (50\%) after a mean of 91 days (SD \pm 24 days). In 15 patients (50\%), no LAMS removal was performed because of death $(n=5)$, significant tissue overgrowth $(n=2)$ or other causes $(n=8)$. Mean follow-up was 298 days (SD \pm 82 days) for all patients and 364 days (SD \pm 82 days) for the patients alive at the end of the study. A total of 15 serious adverse events (SAEs) $(50 \%)$ were reported, including four that were possibly stent-related or procedure-related (13\%). Overall mortality was $23 \%$ (7/30), with 30 -day mortality of $17 \%(5 / 30)$ (find more details in online supplementary methods and results).

\section{COMMENTS}

This study is the first multicentre prospective study on the use of an LAMS for EUS-GBD in high-risk surgical patients with acute cholecystitis. To date, EUS-GBD using an LAMS has been described in eight reports including 30 patients, reporting an overall technical success rate of $93 \% .^{3} 5$ 7-12 This high success rate is most likely an overestimation since the majority of reports included retrospective small case series and case reports, which are prone to publication bias. Technical failures were only reported by de la Serna-Higuera et al who retrospectively evaluated EUS-GBD in 13 patients using the same LAMS as was used in the present study. ${ }^{7}$ These authors reported a technical success rate of $85 \%$, with two technical failures. ${ }^{7}$ In addition, in four patients a second fully covered tubular selfexpandable metal stent (SEMS) was inserted through the LAMS to ensure stent patency and stability, resulting in difficulties with stent placement in 6 of 13 patients $(46 \%)$ in this study. ${ }^{13}$ In our study, technical failures occurred in 3 of 30 patients $(10 \%)$ and technical problems with LAMS deployment in another 2 of 30 patients (7\%), resulting in an overall technical difficulties rate of $17 \%$. However, in all three patients with technical failures, successful endoscopic drainage was ultimately achieved during the same procedure with placement of an additional stent (figures 1 and 2).

In order to improve technical success, refinements of the current LAMS and accessories may improve the results of EUS-GBD. The evolution of the LAMS used in the present study is a new delivery system with electrocautery on the tip, which allows puncture and release of the stent in a singlestep procedure, thus decreasing the number of accessories to be exchanged and consequently potentially reducing the frequency of complications. This newly developed device (Hot Axios, Xlumena, Mountain View, California, USA) has already successfully been used for both gall bladder and PFCs drainage. ${ }^{12} 14$ Furthermore, since the procedure is challenging, even in experienced hands, a learning curve should be anticipated. Because of these considerations, it is our opinion that EUS-GBD should currently only be performed in high-volume experienced centres.

It is known that a mature fistula tract is formed in the porcine model following LAMS placement after a period of $4-5$ weeks. $^{2}$ In order to minimise the risk of recurrent cholecystitis and bile leakage, we decided to leave the LAMS in place for a period of 3 months. A drawback was that we experienced significant tissue overgrowth in three patients $(10 \%)$ at the time of LAMS removal that precluded removal in two patients (figure 2). Although a more significant tissue reaction can be expected after a longer stent dwell time, we hypothesise that stent location, either gastric of duodenal, might also influence the degree of tissue overgrowth. The retroperitoneal location of the duodenum results in a more stable tract to the gall bladder as compared 


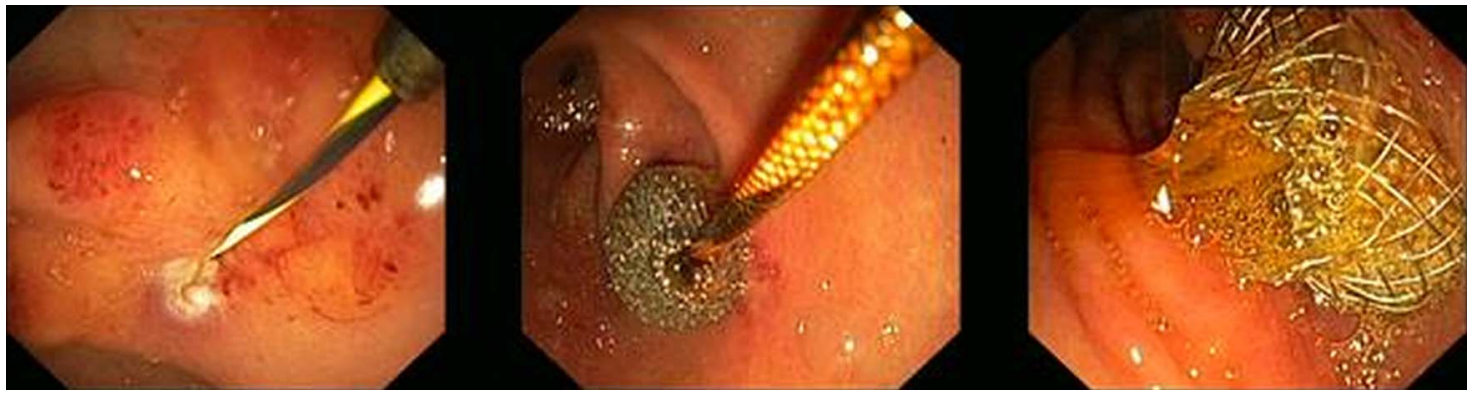

Figure 1 Successful transgastric endoscopic ultrasound-guided gall bladder drainage (EUS-GBD) using a lumen-apposing metal stent (LAMS).

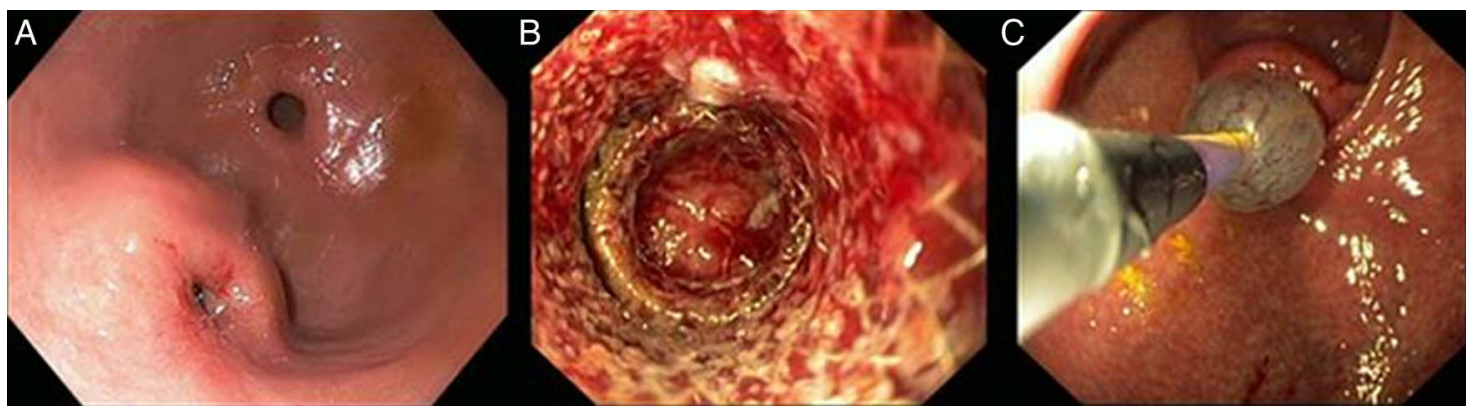

Figure 2 (A) Significant tissue overgrowth of a lumen-apposing metal stent (LAMS) by gastric mucosa after a stent dwell time of 125 days. (B) The LAMS is dilated up to $10 \mathrm{~mm}$ with a balloon, and (C) can be entered and removed inside-out with rat-tooth forceps.

with the stomach, in which more peristaltic movements might result in a more pronounced tissue reaction.

Ultimately, in half of the patients in our study no LAMS removal was performed, mainly due to a poor clinical condition of the patient and/or patients' refusal. In none of these patients, LAMS-related complications were observed during a mean stent dwell time of 364 days. Long-term stenting without stent-related complications, even up to 3 years, has also been reported in other studies on EUS-GBD using SEMS. ${ }^{75-17}$ In the light of these results, leaving stents, either SEMS or LAMS, permanently in place may likely be considered as an alternative treatment option, which avoids the risks and discomfort associated with a repeat procedure for stent removal. Furthermore, although gall bladder drainage is most often intended as a bridge to elective surgery, none of the patients in our study turned out to be eligible for elective cholecystectomy mainly due to their ongoing high surgical risk. In order to reduce the risk of recurrent cholecystitis in these patients, permanent drainage is desirable. The advantage of EUS-GBD compared with PTGBD is that longterm stenting does not require an external drainage catheter, which likely may increase patients' comfort and quality of life. ${ }^{15}$

Safety was closely monitored in our study and all SAEs were reviewed by an independent data safety monitoring board. The 30 -day mortality in our study was $17 \%$, which is comparable with the 30-day mortality or in-hospital death of $15.4 \%$ after PTGBD. In addition, the 7\% stent-related or procedure-related mortality observed in our study is comparable with that of PTGBD (around 4\%). However, the rate of non-fatal SAEs $(n=9,30 \%)$ is substantially higher than reported for PTGBD $(15 \%) .{ }^{18}$ One explanation for this high complication rate could be the relatively poor clinical condition of patients in our study. None of the patients in our study was eligible for elective cholecystectomy, as compared with more than $40 \%$ in studies with patients treated with PTGBD. ${ }^{18}$ Another reason might be our thorough and long-term follow-up with special focus on all types of complications, compared with a great variety of complication registrations in PTGBD studies. ${ }^{18}$ Noteworthy, stent migration was not observed in our study, while this has been reported in up to $7 \%$ after EUS-guided drainage of PFCs using both SEMS and LAMS. ${ }^{6} 19$

In conclusion, we think that EUS-GBD using LAMS is an elegant procedure in high-risk surgical patients with acute cholecystitis when performed by an experienced endoscopist. However, large comparative studies are needed to confirm these promising results, to optimise the technical procedure and to address remaining questions, such as optimal stent dwell time and preferred route of access.

Acknowledgements We would like to thank the members of the Data Safety Monitoring Board for their efforts for this study; Dr ECJ Consten (gastrointestinal surgeon); Dr EW Steyerberg (professor of medical decision making); Dr R. Timmer (gastroenterologist) and Dr EPA Vonken (interventional radiologist).

Contributors DW: literature search, study design, patient inclusion, data collection, data analysis, statistical analysis, data interpretation, drafting of the manuscript and final approval. AYT, TI, MP-M, AL, AS-Y: study design, patient inclusion, data collection, critical revision and final approval of the manuscript. PDS: study design, critical revision and final approval of the manuscript. FPV: study design, patient inclusion, data collection, data interpretation, study supervision, critical revision and final approval of the manuscript.

Competing interests TI: Speaker for Xlumena. AL and MP-M: Consultant for Xlumena.

Ethics approval Ethical Committee and board of all participating centres. Provenance and peer review Not commissioned; internally peer reviewed.

\section{REFERENCES}

1 Jang JW, Lee SS, Song TJ, et al. Endoscopic ultrasound-guided transmural and percutaneous transhepatic gallbladder drainage are comparable for acute cholecystitis. Gastroenterology 2012;142:805-11.

2 Binmoeller KF, Shah J. A novel lumen-apposing stent for transluminal drainage of nonadherent extraintestinal fluid collections. Endoscopy 2011;43:337-42.

3 Moon JH, Choi HJ, Kim DC, et al. A newly designed fully covered metal stent for lumen apposition in EUS-guided drainage and access: a feasibility study (with videos). Gastrointest Endosc 2014;79:990-5. 
4 Shah RJ, Shah JN, Waxman I, et al. Safety and efficacy of endoscopic ultrasound-guided drainage of pancreatic fluid collections with lumen-apposing covered self-expanding metal stents. Clin Gastroenterol Hepatol 2015;13:747-52.

5 Itoi T, Binmoeller KF, Shah J, et al. Clinical evaluation of a novel lumen-apposing metal stent for endosonography-guided pancreatic pseudocyst and gallbladder drainage (with videos). Gastrointest Endosc 2012;75:870-6.

6 Walter D, Will U, Sanchez-Yague A, et al. A novel lumen-apposing metal stent for endoscopic ultrasound-guided drainage of pancreatic fluid collections: a prospective cohort study. Endoscopy 2015;47:63-7.

7 de la Serna-Higuera C, Perez-Miranda M, Gil-Simon P, et al. EUS-guided transenteric gallbladder drainage with a new fistula-forming, lumen-apposing metal stent. Gastrointest Endosc 2013;77:303-8.

8 Monkemuller K, Zabielski M, Dowacz-Grollmann A, et al. Endoluminal transgastric endoscopic anastomosis of the gallbladder using an anchoring self-expanding metal stent. Endoscopy 2013;45(Suppl 2 UCTN):E164-6.

9 Itoi T, Binmoeller K, Itokawa F, et al. Endoscopic ultrasonography-guided cholecystogastrostomy using a lumen-apposing metal stent as an alternative to extrahepatic bile duct drainage in pancreatic cancer with duodenal invasion. Dig Endosc 2013;25(Suppl 2):137-41.

10 Turner BG, Rotman S, Paddu NU, et al. Cholecystoduodenal drainage and gallstone removal in a patient with cholecystitis and unresectable cholangiocarcinoma. Endoscopy 2013;45(Suppl 2 UCTN):E114-15.

11 Teoh AY, Chan AW, Chiu PW, et al. In vivo appearances of gallbladder carcinoma under magnifying endoscopy and probe-based confocal laser endomicroscopy after endosonographic gallbladder drainage. Endoscopy 2014;46(Suppl 1 UCTN):E13-14.
12 Teoh AY, Binmoeller KF, Lau JY. Single-step EUS-guided puncture and delivery of a lumen-apposing stent for gallbladder drainage using a novel cautery-tipped stent delivery system. Gastrointest Endosc 2014;80:1171.

13 Gornals JB, De la Serna-Higuera C, Sanchez-Yague A, et al. Endosonographyguided drainage of pancreatic fluid collections with a novel lumen-apposing stent. Surg Endosc 2013;27:1428-34.

14 Galasso D, Baron TH, Attili F, et al. Endoscopic ultrasound-guided drainage and necrosectomy of walled-off pancreatic necrosis using a metal stent with an electrocautery-enhanced delivery system. Endoscopy 2015;47(Suppl 1):E68.

15 Choi JH, Lee SS, Choi JH, et al. Long-term outcomes after endoscopic ultrasonography-guided gallbladder drainage for acute cholecystitis. Endoscopy 2014;46:656-61.

16 Itoi T, Coelho-Prabhu N, Baron TH. Endoscopic gallbladder drainage for management of acute cholecystitis. Gastrointest Endosc 2010;71: 1038-45.

17 Jang JW, Lee SS, Park do H, et al. Feasibility and safety of EUS-guided transgastric/ transduodenal gallbladder drainage with single-step placement of a modified covered self-expandable metal stent in patients unsuitable for cholecystectomy. Gastrointest Endosc 2011;74:176-81.

18 Winbladh A, Gullstrand P, Svanvik J, et al. Systematic review of cholecystostomy as a treatment option in acute cholecystitis. HPB (Oxford) 2009;11:183-93.

19 Chandran S, Efthymiou M, Kaffes A, et al. Management of pancreatic collections with a novel endoscopically placed fully covered self-expandable metal stent: a national experience (with videos). Gastrointest Endosc 2015;81:127-35. 